\title{
THE RECIPROCAL OF A FOURIER SERIES ${ }^{1}$
}

\section{R. J. DUFFIN}

Edrei and Szegö [1] have posed the following problem: Given the Fourier coefficients of a function $G(x)$ find the Fourier coefficients of the reciprocal of the function without actually evaluating $G(x)$. They were able to solve this problem in the case that $G(x) \geqq 0$. Unfortunately this restriction eliminates the interesting case of complex-valued functions such as those which arise in Laurent series.

In this note it is shown possible to obtain a solution without the restriction, $G(x) \geqq 0$. This is achieved by first treating the more general problem of finding the coefficients of $F(x) / G(x)$, given the coefficients of $F(x)$ and $G(x)$.

Edrei and Szegö confine attention to classical Fourier series. Their problem is treated here for arbitrary orthogonal series expansions. ${ }^{2}$

Let $\theta_{j}(x), j=1,2, \cdots$, be a sequence of bounded orthonormal functions in some region $R$ of a space $S$. Thus

$$
\int_{R} \theta_{j}(x) \theta_{k}^{*}(x) d x=\delta_{j k}
$$

where the asterisk denotes the complex conjugate. The Fourier coefficients of a function $F(x) \in L$ are denoted by $f_{j}$ and are given by the integral

$$
f_{j}=\int_{R} F(x) \theta_{j}^{*}(x) d x, \quad j=1,2, \cdots
$$

If $f_{j}=0$ for all $j$ it is assumed that $F(x)=0$ almost everywhere. In other words the orthonormal sequence is closed in $L$.

Problem 1. Given the Fourier coefficients $f_{n}$ and $g_{n}$ of two functions $F(x)$ and $G(x)$ find the Fourier coefficients $h_{n}$ of the function $H(x)$ $=F(x) / G(x)$.

A related question concerns a direct determination of $H(x)$ without first finding $F(x)$ and $G(x)$. This may be stated as

PROBLEM 2. In terms of $f_{n}$ and $g_{n}$ find expansion coefficient sets $\left(\rho_{1}^{(m)}, \rho_{2}^{(m)}, \cdots, \rho_{m}^{(m)}\right)$ for $m=1,2, \cdots$ such that

$$
\lim _{m \rightarrow \infty} \int_{R}\left|H(x)-\sum_{k=1}^{m} \rho_{k}^{(m)} \theta_{k}(x)\right| d x=0 .
$$

Received by the editors April 28, 1961 and, in revised form, December 2, 1961.

1 Prepared under Contract DA-36-061 ORD-490, U. S. Army Research Office.

2 This generalization was suggested to the writer by David Moskovitz. 
Given a solution of Problem 2 then

$$
\lim _{m \rightarrow \infty} \int_{R}\left[H(x)-\sum_{k=1}^{m} \rho_{k}^{(m)} \theta_{k}(x)\right] \theta_{j}^{*}(x) d x=0 .
$$

This is seen to give

$$
h_{j}=\lim _{m \rightarrow \infty} \rho_{j}^{(m)}
$$

and consequently the solution of Problem 2 also furnishes a solution of Problem 1. (In the following $\rho_{j}^{(m)}$ is denoted by $\rho_{j}$.)

Problem 3. The solution of Problem 2 is sought but given the "Fourier matrix"

$$
g_{j k}=\int_{R} G(x) \theta_{j}^{*}(x) \theta_{k}(x) d x
$$

instead of the Fourier coefficients $g_{j}$. In other words find $h_{n}$ in terms of $f_{n}$ and $g_{j k}$. The following formal considerations indicate the close relationship between Problem 2 and Problem 3. Let

$$
\beta_{j k r}=\int_{R} \theta_{j}^{*}(x) \theta_{k}(x) \theta_{r}(x) d x .
$$

Then

$$
\theta_{j}^{*}(x) \theta_{k}(x)=\sum_{r=1}^{\infty} \beta_{j k r} \theta_{r}^{*}(x)
$$

This relation is multiplied by $G(x)$ and integrated termwise to yield

$$
g_{j k}=\sum_{r=1}^{\infty} \beta_{j k r} g_{r} .
$$

Thus if (9) is valid then a solution of Problem 3 will yield a solution of Problem 2.

Theorem 1. Let $G(x) \geqq 0, G(x) \in L, 1 / G(x) \in L$, and $F^{2}(x) / G(x) \in L$. Then for each integer $m$ the equations

$$
f_{j}=\sum_{k=1}^{m} g_{j k} \rho_{k}, \quad j=1, \cdots, m,
$$

may be solved for $\rho_{1}, \rho_{2}, \cdots, \rho_{m}$ and these are expansion coefficient sets for $H(x)=F(x) / G(x)$ and this solves Problem 3 . 
Proof. Since $F=H G^{1 / 2} G^{1 / 2}$ the Schwarz inequality gives

and

$$
\left(\int|F| d x\right)^{2} \leqq \int\left|H^{2} G\right| d x \int|G| d x
$$

$$
\left(\int|H| d x\right)^{2} \leqq \int\left|H^{2} G\right| d x \int\left|G^{-1}\right| d x .
$$

This proves that $F \in L$ and $H \in L$. Then it is seen that the following integral exists for any choice of the constants $\rho_{j}$ :

$$
E=\int\left|H(x)-\sum_{1}^{m} \rho_{k} \theta_{k}(x)\right|^{2} G(x) d x .
$$

Since $G(x) \geqq 0$ this may be written in the form

$$
E=\int\left|H G^{1 / 2}-\sum_{1}^{m} \rho_{k} \theta_{k} G^{1 / 2}\right|^{2} d x
$$

This leads to consideration of the relation

$$
\int U(x) \theta_{j}^{*}(x) G^{1 / 2} d x=0, \quad j=1,2, \cdots,
$$

where $U(x) \in L^{2}$. Then $U G^{1 / 2} \in L$ and since the sequence $\theta_{j}$ is closed in $L$ relation (13) implies $U G^{1 / 2}=0$ almost everywhere. Thus $U=U G^{1 / 2} G^{-1 / 2}=0$ almost everywhere. This proves that the sequence $\theta_{j} G^{1 / 2}$ is closed in $L^{2}$.

Closure in $L^{2}$ implies completeness in $L^{2}$ so it is possible to make $E$ arbitrarily small. This may be accomplished by choosing, for each $m$, the set $\left(\rho_{1}, \cdots, \rho_{m}\right)$ which minimizes $E$. As is well known this optimal choice is determined by the orthogonality equations

$$
0=\int\left(H-\sum_{1}^{m} \rho_{k} \theta_{k}\right) \theta_{j}^{*} G d x, \quad j=1,2, \cdots, m .
$$

Since $H G=F$ it is apparent that these are precisely equations (10) of Theorem 1.

By the Schwarz inequality

$$
\left(\int\left|H-\sum_{1}^{m} \rho_{k} \theta_{k}\right| d x\right)^{2} \leqq E \int\left|G^{-1}\right| d x .
$$

The left side approaches zero if $E$ approaches zero. This shows that the optimal choice of $\rho_{j}$ leads to the satisfaction of condition (3) of Problem 2 and completes the proof. 
Theorem 2. Suppose $F(x) \in L^{2}$ and $1 / F(x) \in L^{2}$. Let

$$
\begin{aligned}
\beta_{j k r s} & =\int_{R} \theta_{j}^{*}(x) \theta_{k}(x) \theta_{r}^{*}(x) \theta_{s}(x) d x, \\
g_{j k} & =\sum_{1}^{\infty} \sum_{1}^{\infty} \beta_{j k r s} f_{r}^{*} f_{s} .
\end{aligned}
$$

Then the equations

$$
f_{j}=\sum_{1}^{m} g_{j k} \rho_{k}, \quad j=1,2, \cdots, m,
$$

may be solved for $\rho_{1}, \rho_{2}, \cdots, \rho_{m}$. For $m=1,2, \cdots$ these solutions form $a$ set of expansion coefficients of the function $H(x)=1 / F^{*}(x)$.

Proof. Define $G(x)=F(x) F^{*}(x)$ then $H(x)=F(x) / G(x)$. Then $G(x) \geqq 0, G(x) \in L,(G(x))^{-1} \in L$, and $H^{2}(x) G(x) \in L$. Thus the conditions of Theorem 1 are satisfied. Substituting $G=F F^{*}$ in relation (6) for the Fourier matrix gives

$$
g_{i k}=\int \theta_{j}^{*} \theta_{k} F^{*} F d x .
$$

The series $\sum_{1}^{\infty} f_{r}^{*} \theta_{r}^{*}$ and $\sum_{1}^{\infty} f_{s} \theta_{s}$ converge in $L^{2}$ mean to $F^{*}$ and $F$. Therefore it is permissible to substitute these series in (19) and to integrate termwise. This justifies (17); the proof then follows from Theorem 1.

Theorem 3. Let $G(x) \geqq 0, G(x) \in L, 1 / G(x) \in L$, and $F^{2}(x) / G(x) \in L$ in the interval $0 \leqq x \leqq 1$. The Fourier coefficients are now defined as

$$
f_{j}=\int_{0}^{1} F(x) \exp (-2 \pi i j x) d x, \quad j=0, \pm 1, \cdots .
$$

Then the equations

$$
f_{j}=\sum_{-m}^{m} g_{j-k} \rho_{k}, \quad j=-m, \cdots, m,
$$

may be solved for $\rho_{-m}, \cdots, \rho_{m}$ and

$$
\int_{0}^{1}\left|F(x) / G(x)-\sum_{-m}^{m} \rho_{k} \exp (2 \pi i k x)\right| d x \rightarrow 0 \text { as } m \rightarrow \infty .
$$

Proof. Theorem 1 is specialized with the sequence $\theta_{j}(x)$ being the sequence $\exp (2 \pi i j x)$ suitably reordered. It is then seen that

$$
g_{j k}=g_{j-k}
$$


and thus equation (10) becomes equation (21). The proof of relation (22) then follows from Theorem 1.

Theorem 4. Suppose $F(x) \in L^{2}$ and $1 / F(x) \in L^{2}$ on the interval $0 \leqq x \leqq 1$. Let $f_{j}$ denote the Fourier coefficients of $F(x)$ relative to the orthonormal sequence exp $(2 \pi i j x)$. Let

$$
g_{j}=\sum_{-\infty}^{\infty} f_{j+r} f_{r}^{*}
$$

Then the equations

$$
f_{-j}^{*}=\sum_{-m}^{m} g_{j-k} \alpha_{k}
$$

may be solved for $\alpha_{-m}, \cdots, \alpha_{m}$ and

$$
\int_{0}^{1}\left|(F(x))^{-1}-\sum_{-m}^{m} \alpha_{k} \exp (2 \pi i k x)\right| d x \rightarrow 0 \text { as } m \rightarrow \infty .
$$

Proof. First Theorem 2 is applied to obtain $H=1 / F^{*}$. It is seen from (16) that $\beta_{j k r s}=1$ if $s=j-k+r$ and $\beta_{j k r s}=0$ otherwise. Thus (17) gives

$$
g_{j k}=g_{j-k}=\sum_{-\infty}^{\infty} f_{j-k+r} f_{r}^{*}
$$

Then (18) is

$$
f_{j}=\sum_{-m}^{m} g_{j-k} \rho_{k} .
$$

Taking the complex conjugate of this relation gives

$$
f_{-j}^{*}=\sum_{-m}^{m} g_{k-j}^{*} \rho_{-k}^{*}
$$

But $g_{-k}^{*}=g_{k}$ and $\rho_{-k}^{*}=\alpha_{k}$ so this proves (25).

The questions studied in this note originated from the need for an algorithm which would give the reciprocal of a Laurent series. (In this connection it is worth noting that there is a simple algorithm for the reciprocal of a Taylor series.) An algorithm for the reciprocal of a Laurent series is stated in the following Corollary of Theorem 4.

Corollary. Consider the Laurent series

$$
S(z)=\sum_{-\infty}^{\infty} a_{n} z^{n}
$$


which is assumed to converge when the complex variable $z$ is in the region $D$ defined by the relation $r_{1}<|z|<r_{2}$. If $S(z)$ does not vanish in $D$ it follows, of course, that the reciprocal $T(z)=1 / S(z)$ is a Laurent series in D. Thus

$$
T(z)=\sum_{-\infty}^{\infty} c_{n} z^{n} .
$$

Let coefficients $b_{j}$ be defined as

$$
b_{j}=\sum_{-\infty}^{\infty} a_{j+r} a_{r}^{*}
$$

Then the equations

$$
a_{-j}^{*}=\sum_{-m}^{m} b_{j-k} C_{k}^{(m)}, \quad j=-m, \cdots, m,
$$

may be solved for $C_{-m}^{(m)}, \cdots, C_{m}^{(m)}$. Let

$$
T_{m}(z)=\sum_{-m}^{m} C_{k}^{(m)} z^{k}
$$

Then as $m \rightarrow \infty$ the sequence $T_{m}(z)$ converges uniformly to $T(z)$ in any closed region contained in $D$. Moreover, $C_{\boldsymbol{k}}^{(m)} \rightarrow c_{k}$ as $m \rightarrow \infty$.

Proof. The function $F(x)=S\left(r e^{2 \pi i x}\right)$ satisfies the condition of Theorem 4 for any value of $r$ in the range $r_{1}<r<r_{2}$. The Fourier coefficients of $F$ are given by $f_{j}=r^{j} a_{j}$. Defining $b_{j}$ and $C_{j}^{(m)}$ by the relations $g_{j}=r^{i} b_{j}$ and $\alpha_{j}=r^{i} C_{j}^{(m)}$ it results that relations (24) and (25) yield relations (29) and (30). It follows that $T_{m}$ converges in mean to $T$ for $|z|=r$. Likewise $T_{m}$ converges in mean for $|z|=r^{\prime}$ where $r_{1}<r<r^{\prime}<r_{2}$. Then by a standard theorem from complex function theory it follows that $T_{m}$ converges uniformly to $T$ for $r<|z|<r^{\prime}$. This is seen to complete the proof of the Corollary.

\section{REFERENCE}

1. A. Edrei and G. Szegö, $A$ note on the reciprocal of a Fourier series, Proc. Amer. Math. Soc. 4 (1953), 323-329.

Carnegie Institute of Technology 